11,09

\title{
Оптические свойства пленок ниобата бария стронция SBN61
}

\author{
(C) В.Б. Широков ${ }^{1,2}$, А.В. Павленко ${ }^{1,2}$, Д.В. Стрюков ${ }^{1,2}$, Ю.В. Ревинский \\ ${ }^{1}$ Южный научный центр РАН, \\ Ростов-на-Дону, Россия \\ 2 Южный федеральный университет, \\ Ростов-на-Дону, Россия \\ E-mail: shirokov-vb@rambler.ru \\ (Поступила в Редакцию 6 июля 2017 г. \\ В окончательной редакции 12 декабря 2017 г.)
}

\begin{abstract}
Методом высокочастотного RF-напыления в атмосфере кислорода получены гетероэпитаксиальные пленки $\mathrm{Sr}_{0.61} \mathrm{Ba}_{0.39} \mathrm{Nb}_{2} \mathrm{O}_{6}$ на подложках $\mathrm{MgO}$ (001). Ориентация пленок относительно подложки исследована по данным измерений рентгеновской дифракции. По результатам оптических измерений спектров пропускания определены параметры дисперсии диэлектрической проницаемости в видимом диапазоне. Установлено, что полученные пленки, по сравнению с монокристаллом, имеют бо́льшую ширину щели запрещенной зоны и меньший показатель преломления.
\end{abstract}

Работа выполнена в рамках государственного задания ЮНЦ РАН (тема № госрегистрации 01201354247).

DOI: 10.21883/FTT.2018.05.45801.228

\section{1. Введение}

Твердые растворы ниобата стронция-бария $\mathrm{Sr}_{x} \mathrm{Ba}_{1-x} \mathrm{Nb}_{2} \mathrm{O}_{6}(\mathrm{SBN})$ [1] привлекают большое внимание исследователей благодаря своим электрооптическим [2], пироэлектрическим [3] и пьезоэлектрическим [4] свойствам, которые открывают большие перспективы их использования в устройствах микроэлектроники. Кристаллы SBN являются привлекательными материалами для новых оптико-электронных устройств. Особый интерес вызывают высокая фоторефрактивная чувствительность и большой электрооптический коэффициент $r_{33}$ [5], что позволяет применять его для современных устройств оптической обработки данных [6], оптических накопителей [7] и оптического сопряжения [8].

Твердые растворы SBN при концентрации стронция $x$ от 0.2 до 0.8 принадлежат к структуре тетрагональной вольфрамовой бронзы [9]. Пространственная группа структуры - P4bm, а элементарная ячейка содержит пять формульных единиц $(\mathrm{Sr}, \mathrm{Ba}) \mathrm{Nb}_{2} \mathrm{O}_{6}$ [10]. Параметры ячейки для состава $\mathrm{Sr}_{0.61} \mathrm{Ba}_{0.39} \mathrm{Nb}_{2} \mathrm{O}_{6}$ (SBN61) $a=12.456(1) \AA$ и $c=3.936(2) \AA[11]$. В структуре $\mathrm{SBN}$ имеются два неэквивалентных $\mathrm{NbO}_{6}$ октаэдра, в центре которых расположены катионы ниобия. Катионы стронция заполняют каналы квадратного сечения. Каналы больших размеров (пятиугольного сечения) заполняют катионы и стронция и бария $[12,13]$. При изменении концентрационного соотношения $\mathrm{Ba} / \mathrm{Sr}$ меняются параметры решетки [5,14], происходит перераспределение заполнения каналов атомами $\mathrm{Ba}$ и $\mathrm{Sr}[10,12,13]$. При этом претерпевают изменения диэлектрические $[5,15]$, оптические [16] и электрооптические $[17,18]$ свойства. Перспективным материалом для применения являются тонкие пленки SBN. Наиболее интересны гетероэпитаксиальные пленки, которые получаются методами лазерной абляции (PLD) [19-25] и высокочастотным распылением [26,27] мишеней.

В данной работе исследуются свойства тонких пленок SBN61, синтезированных методом высокочастотного RFнапыления в атмосфере кислорода на монокристаллических подложках $\mathrm{MgO}$ (001) среза. Для адекватного учета интерференционных эффектов при оптических измерениях использованы подложки с двухсторонней полировкой.

\section{2. Методы получения и исследования объектов}

Напыление пленок SBN61 на монокристаллические подложки $\mathrm{MgO}$ (001) выполнено на установке Плазма50-СЭ по методике, описанной в [28]. Структурное совершенство пленок, параметры элементарной ячейки, а также ориентационные соотношения между пленкой и подложкой устанавливались методами рентгеновской дифрактометрии на дифрактометре „ДРОН-3М“ $\left(\mathrm{Cu} K_{\alpha}\right.$-излучение), оснащенного интенсиметром ИР-2, с использованием симметричного и асимметричного сканирования брэгговских отражений. Топография поверхности пленок SBN61 анализировалась на атомносиловом микроскопе NTEGRA PRIMA фирмы HTМДТ (Россия). Измерения коэффициентов пропускания пленок SBN61 в оптическом диапазоне длин волн 190-1100 nm (1.1-6.5 eV) выполнены на спектрометре Backman Du800.

\section{3. Экспериментальные результаты}

В результате рентгенодифракционного сканирования в исследованных пленках не выявлено примесных фаз. 
Рентгенограммы $\theta / 2 \theta$ сканирования исследованных гетероструктур SBN61/MgO (рис. 1) представляют собой набор $(00 l)$ отражений от плоскостей пленки и подложки. Это указывает на сонаправленность осей [001] пленки и [001] подложки. Из полученных рентгенограмм определены одинаковые параметры решетки $a=12.37(3) \AA, c=3.953(1) \AA$; для пленок SBN61 35 и $115 \mathrm{~min}$ соответственно. Толщина пленок, по результатам предварительных исследований, на подложкахсколах линейно связана со временем напыления. Для выбранного режима напыления одна минута напыления соответствует толщине порядка $6 \mathrm{~nm}$.

Доказательством эпитаксиального роста пленок SBN61 на подложке $\mathrm{MgO}$ является сопоставление $\varphi$ сканирования рефлексов (221) пленки и (113) подложки (рис. 2, $a, b)$. На $\varphi$ сканировании отражения (221)

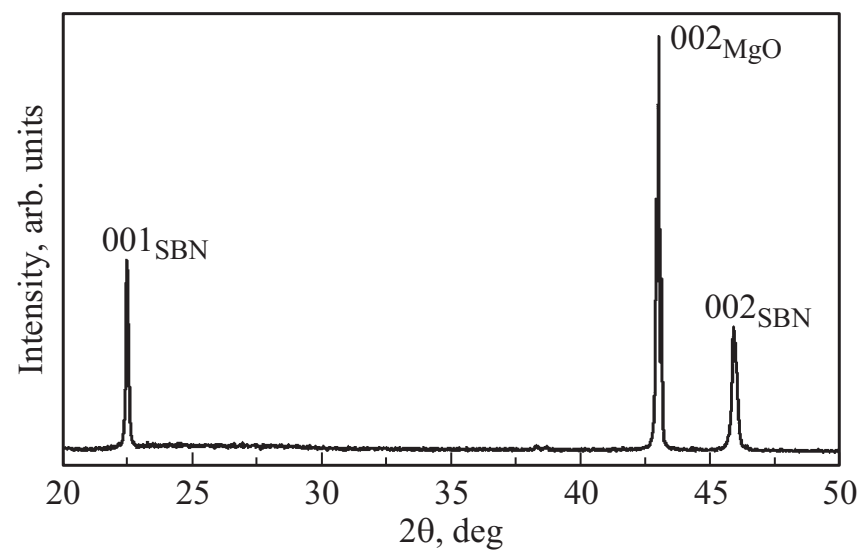

Pис. 1. Рентгенограмма $\theta / 2 \theta$ пленки $\mathrm{SBN} 61 / \mathrm{MgO}$ толщиной $202 \mathrm{~nm}(35 \mathrm{~min})$.

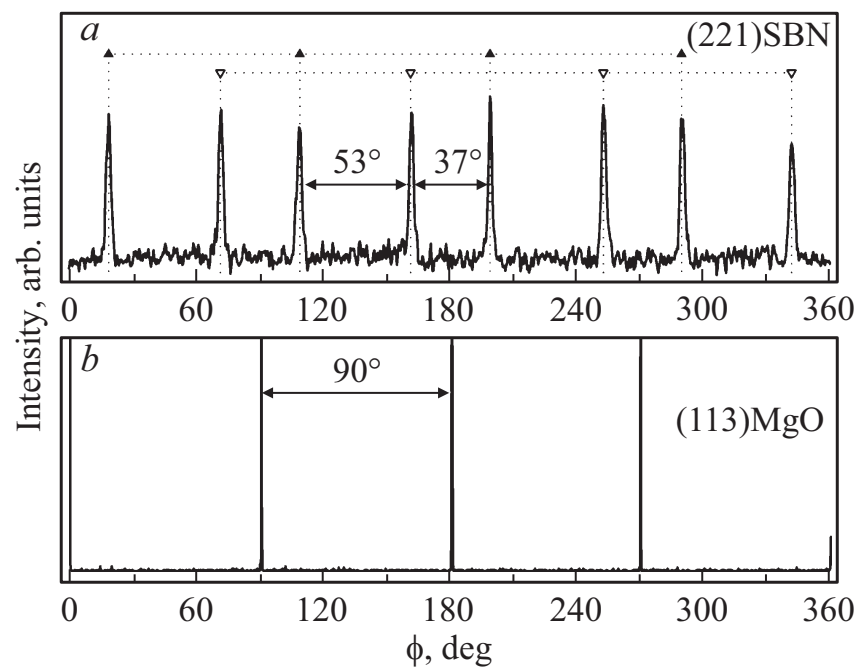

Рис. 2. $a)-\varphi$ сканирование рефлекса (221) пленки SBN61, где заполненные символы соответствуют ориентационному домену с $+18.43^{\circ}$ между осями 100 пленки и 100 подложки, пустые символы соответствуют $\left.-18.43^{\circ} ; b\right)-\varphi$ сканирование рефлекса (113) подложки $\mathrm{MgO}$.

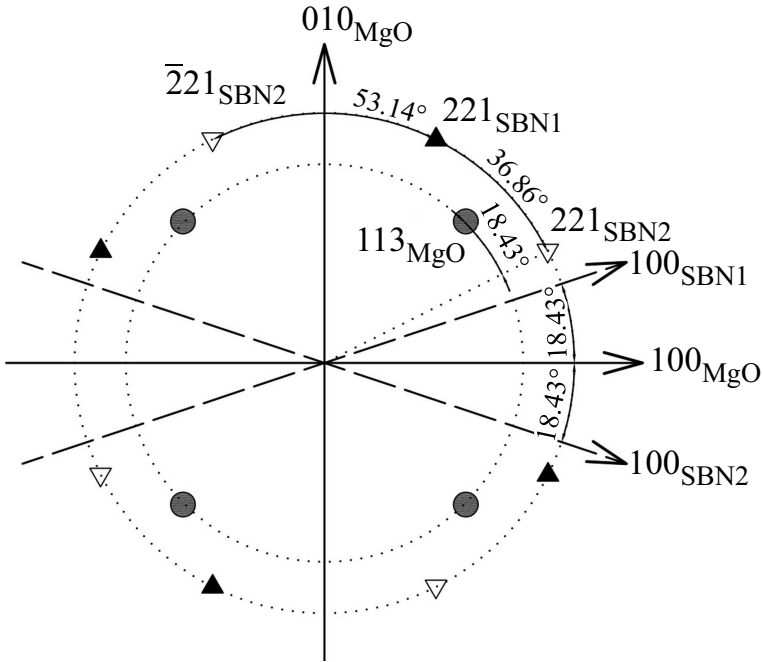

Рис. 3. Схема относительной ориентации рефлексов 221 пленки SBN и 113 подложки $\mathrm{MgO}$, где заполненные треугольники (SBN1) соответствуют фазе с $+18.43^{\circ}$ между осями 100 пленки и 100 подложки, пустые треугольники (SBN2) соответствует $-18.43^{\circ}$, а заполненные кружки соответствуют отражениям 113 подложки $\mathrm{MgO}$.

пленки присутствует 8 максимумов, такое распределение максимумов соответствует двум ориентационным доменам SBN61 с углом между ними в $36.87^{\circ}$. Угловые положения отражений (113) подложки находятся на равном удалении $\left(18.43^{\circ}\right)$ от двух ближайших максимумов отражений (221) пленки, причем эти максимумы относятся к разным ориентационным доменам. Таким образом, получаем две симметричные относительно подложки ориентации с уголом $\pm 18.43^{\circ}$ между осями [110] каждого из ориентационных доменов пленки и осью [110] подложки. Эпитаксиальные соотношения для исследованных пленок приведены в табл. 1 и графически проиллюстрированы на рис. 3. Вертикальная разориентировка (ширина на половине высоты кривой качания) слоев пленки составляет порядка $0.9^{\circ}$. Анализ полуширин асимметричных отражений от слоев пленки при $\varphi$ сканировании, показал, что азимутальная разориентировка составляет менее $0.4^{\circ}$.

Топография поверхности полученных пленок SBN61 представлена на рис. 4. Среднеквадратичная шероховатость поверхности пленок 1.9 и $4.0 \mathrm{~nm}$ для времен напыления 35 и $115 \mathrm{~min}$ соответственно. С учетом

Таблица 1. Эпитаксиальные соотношения для исследованных пленок SBN61 на монокристаллических подложках MgO

\begin{tabular}{c|c}
\hline $\operatorname{SBN} 1\left(+18.4^{\circ}\right)$ & $\operatorname{SBN} 2\left(-18.4^{\circ}\right)$ \\
\hline$[001]_{\mathrm{film}} \|[001]_{\mathrm{MgO}}$ & {$[001]_{\mathrm{film}} \|[001]_{\mathrm{MgO}}$} \\
{$[010]_{\mathrm{film}} \|[310]_{\mathrm{MgO}}$} & {$[010]_{\mathrm{film}} \|[3 \overline{1} 0]_{\mathrm{MgO}}$} \\
{$[100]_{\mathrm{film}} \|[\overline{130}]_{\mathrm{MgO}}$} & {$[100]_{\mathrm{film}} \|[130]_{\mathrm{MgO}}$}
\end{tabular}



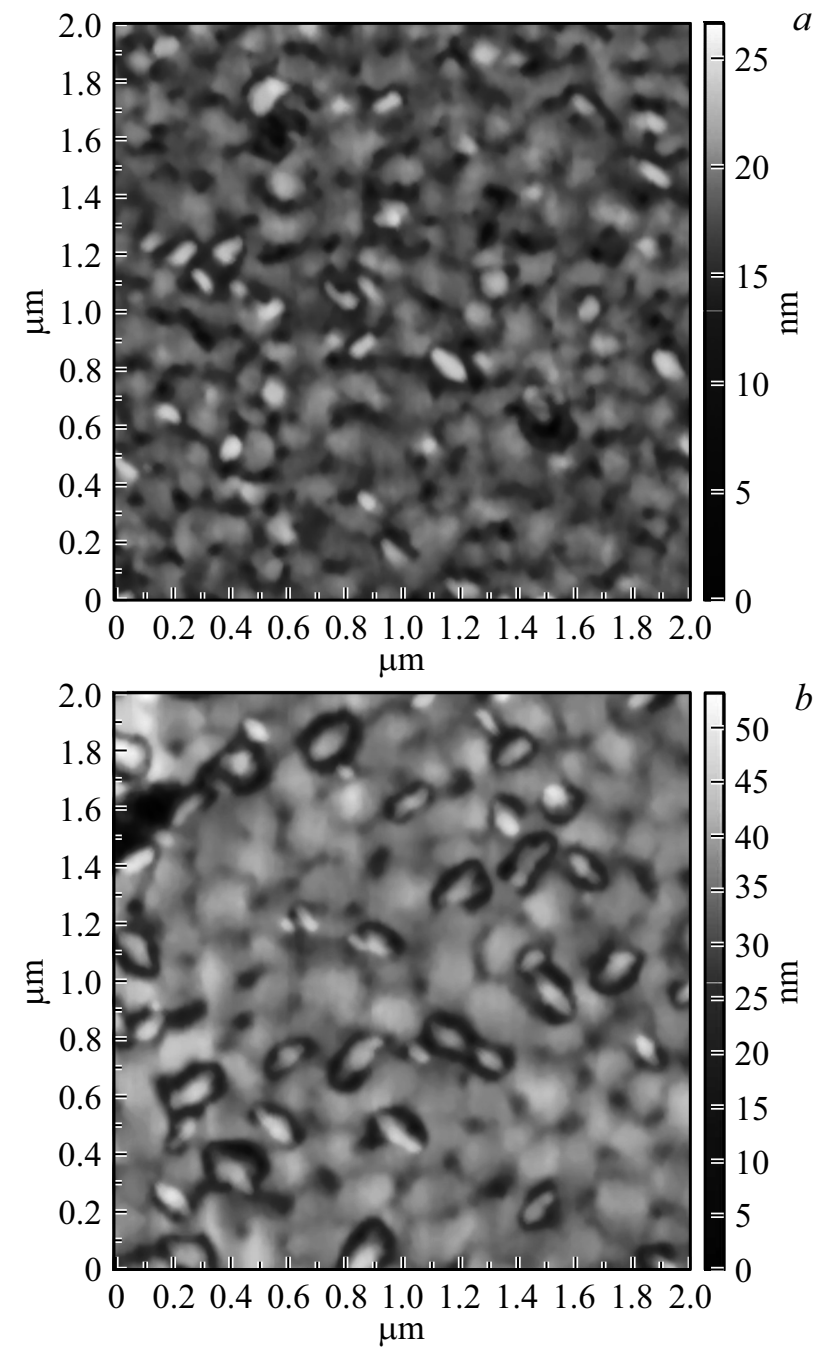

Рис. 4. АCM изображение поверхности пленок SBN61 с временем (толщиной) напыления $35 \mathrm{~min}(a)$ и $115 \mathrm{~min}(b)$.

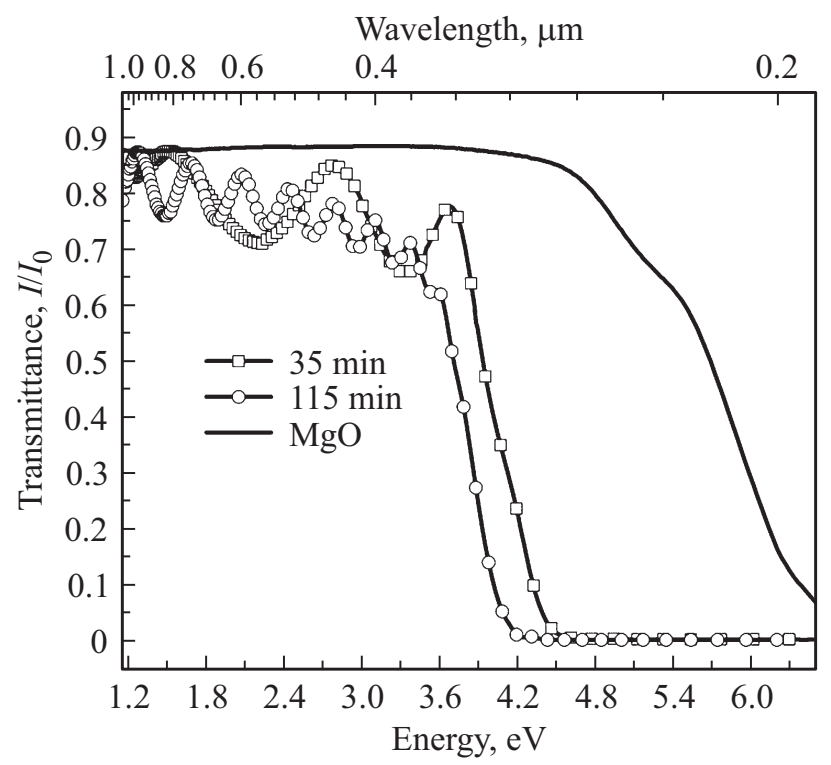

Pис. 5. Спектры пропускания пленок SBN61 на подложке $\mathrm{MgO}$. результатов измерений пленок с меньшим временем напыления наблюдается увеличение шероховатости при увеличении времени напыления.

Спектры пропускания подложки $\mathrm{MgO}$ и гетероструктур SBN61/MgO в оптическом диапазоне длин волн 190-1100 nm (1.1-6.5 eV) представлены на рис. 5. Как видно из рисунка, граница спектра (край поглощения) пленок по энергиям уменьшается с увеличением их толщины. Для обработки спектров пропускания использована формула для пропускания тонкой пленки на толстой подложке при падении излучения по нормали к плоскости пленки [29]

$$
T=\frac{n_{S}\left|t_{123}\right|^{2}\left|t_{31}\right|^{2} \exp \left(-2 \alpha^{\prime \prime}\right)}{1-\left|r_{321}\right|^{2}\left|r_{31}\right|^{2} \exp \left(-4 \alpha^{\prime \prime}\right)},
$$

где $\alpha^{\prime \prime}=2 \pi k_{S} \frac{d}{\lambda}, n_{S}, k_{S}$ - действительная и мнимая части показателя преломления подложки, $d-$ толщина подложки,

$$
\begin{aligned}
& t_{123}=\frac{t_{12} t_{23} \exp (i \delta)}{1+r_{12} r_{23} \exp (2 i \delta)}, \quad r_{321}=\frac{r_{32}+r_{21} \exp (i \delta)}{1+r_{32} r_{21} \exp (2 i \delta)}, \\
& r_{i j}=\frac{n_{j}+i k_{j}-1}{n_{i}+i k_{i}+1}, \quad t_{i j}=1-r_{i j} \delta=2 \pi\left(n_{j}+i k_{j}\right) \frac{h}{\lambda} .
\end{aligned}
$$

Индекс 1 относится к воздуху: $n_{1}=1, k_{1}=0$, индекс 2 соответствует пленке: $n_{2}=n_{f}, k_{2}=k_{f}$, а индекс 3 нумерует показатель преломления подложки: $n_{3}=n_{S}$, $k_{3}=k_{S}$.

Дисперсионная формула для диэлектрической проницаемости нами взята в виде суммы осцилляторов с учетом релаксации [30,31] при выполнении правила симметрии $\varepsilon(-\omega)=\varepsilon^{*}(\omega)$ [32]

$$
\varepsilon=\varepsilon_{\infty}+\sum_{i=0}^{N} \frac{f_{i}-i f_{R, i} E}{E_{i}^{2}-E^{2}-i \gamma_{i} E},
$$

где $E_{i}, \gamma_{i}, f_{i}-$ собственная энергия, затухание и интенсивность $i$-го осциллятора соответственно, $f_{R, i}-$ параметр, характеризующий состояние осциллятора под действием падающего излучения. Параметры в (3) взяты в размерном виде для удобства сравнения осцилляторов разной энергии. Для действительной и мнимой частей диэлектрической проницаемости из (3) имеем

$$
\begin{gathered}
\varepsilon^{\prime}=\varepsilon_{\infty}+\sum_{i=0}^{N} \frac{f_{i}\left(E_{i}^{2}-E^{2}\right)+\gamma_{i} f_{R, i} E^{2}}{\left(E_{i}^{2}-E^{2}\right)^{2}+\gamma_{i}^{2} E^{2}}, \\
\varepsilon^{\prime \prime}=\sum_{i=0}^{N} \frac{E\left(\gamma_{i} f_{i}-f_{R, i}\left(E_{i}^{2}-E^{2}\right)\right)}{\left(E_{i}^{2}-E^{2}\right)^{2}+\gamma_{i}^{2} E^{2}},
\end{gathered}
$$

где действительная и мнимая части диэлектрической проницаемости определены в (4).

Показатель преломления, используемый в вышеприведенных формулах (1) и (2), связан с диэлектрической 

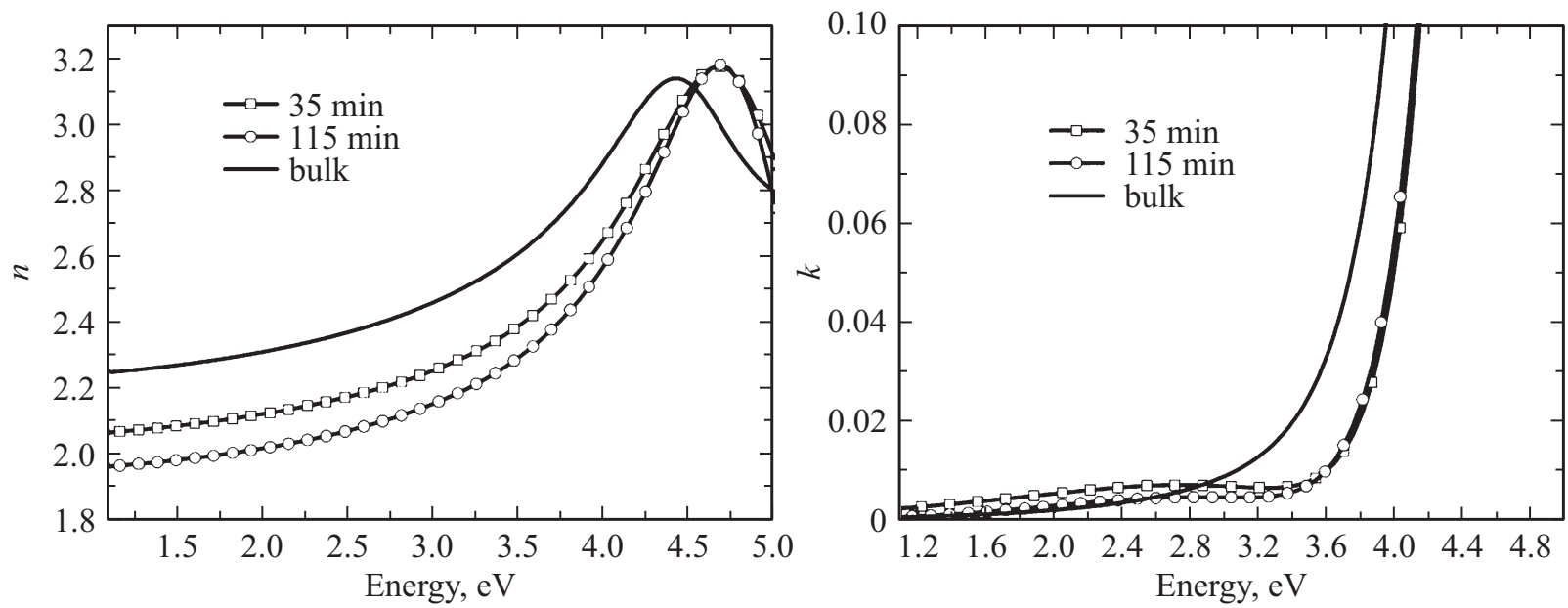

Рис. 6. Действительная часть $n$ (индекс рефракции) и мнимая часть $k$ (коэффициент экстинкции) показателя преломления тонких пленок SBN61 разной толщины.
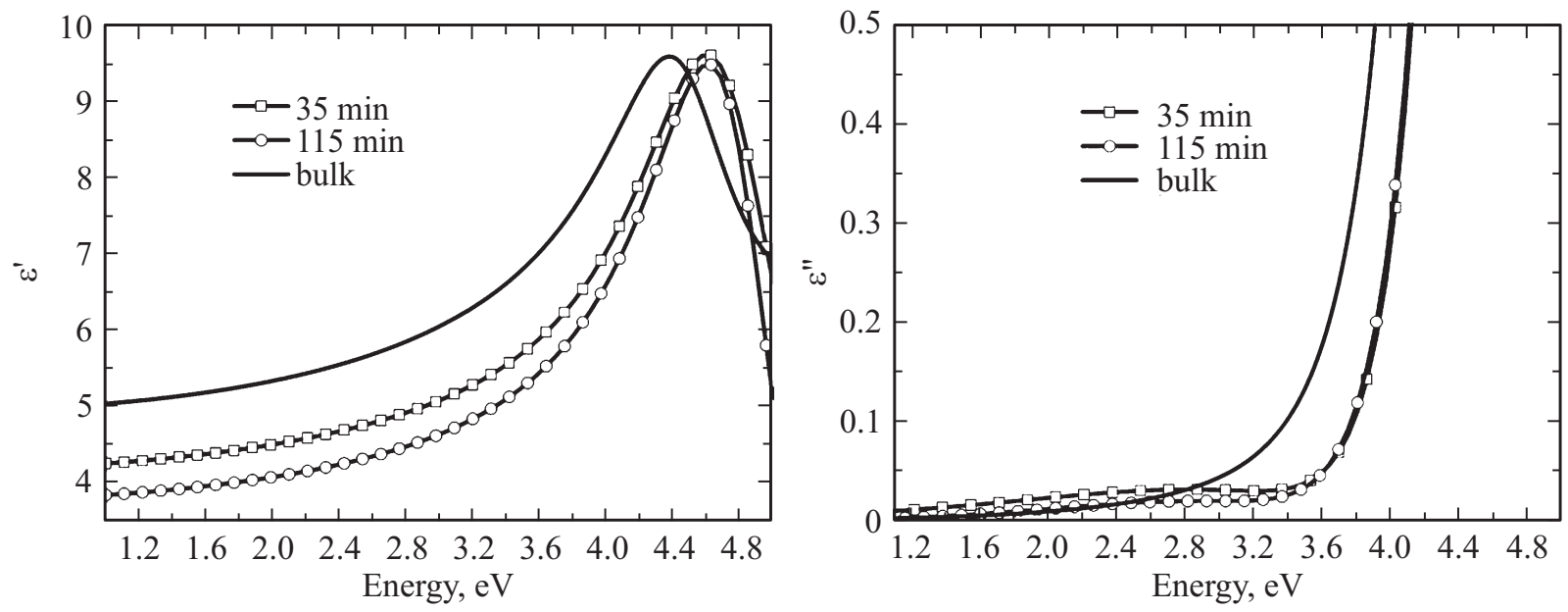

Рис. 7. Действительная часть $\varepsilon^{\prime}$ (слева) и мнимая часть $\varepsilon^{\prime \prime}$ (справа) диэлектрической проницаемости тонких пленок SBN61 разной толщины.

проницаемостью следующим образом

$$
\begin{aligned}
& n=\frac{1}{2} \sqrt{2 \varepsilon^{\prime}+2 \sqrt{\left(\varepsilon^{\prime}\right)^{2}+\left(\varepsilon^{\prime \prime}\right)^{2}}}, \\
& k=\frac{\varepsilon^{\prime \prime}}{\sqrt{2 \varepsilon^{\prime}+2 \sqrt{\left(\varepsilon^{\prime}\right)^{2}+\left(\varepsilon^{\prime \prime}\right)^{2}}}} .
\end{aligned}
$$

Экспериментальные данные (рис. 2) обрабатывались по методу наименьших квадратов с использованием формул (1)-(5). Параметры осцилляторов для подложки $\mathrm{MgO}$ были определены ранее [30,31] и здесь не приводятся. Дисперсионная формула диэлектрической проницаемости для пленки SBN61 взята в виде двух осцилляторов из следующих соображений. Согласно расчетам из первых принципов, край поглощения в ниобатах с кислородным октаэдрическим окружением, формируется электронными переходами между зонами, образованными состояниями $\mathrm{O}^{2 p}$ и $\mathrm{Nb}^{4 d}$. Энергии этих межзонных оптических переходов порядка 4.4 и $5.3 \mathrm{eV}$ (см., например, [33]). При вычислениях, толщина пленки входила в число варьируемых параметров. Полученная таким образом толщина пленки - оптическая толщина. Вычисления проводились до достижения сред-

Таблица 2. Параметры осцилляторов (5) для пленок SBN61 и подложки $\mathrm{MgO}$, вычисленные по спектрам пропускания (рис. 5). (Обозначение параметров согласно формуле (4), $\sigma$ - среднеквадратичное отклонение коэффициента пропускания (1) от экспериментальных значений.)

\begin{tabular}{c|c|c|c|c|c|l}
\hline SBN61 & $h$ & $\varepsilon_{\infty}$ & $E_{i}(\mathrm{eV})$ & $\gamma_{i}$ & $f_{i}$ & $f_{R, i}$ \\
\hline $35 \mathrm{~min}$ & $202 \mathrm{~nm}$ & 1.28 & 4.78 & 1.09 & 19.97 & 6.85 \\
$\sigma=0.7 \%$ & & & 5.91 & 4.85 & 70.74 & 0.6 \\
$115 \mathrm{~min}$ & $736 \mathrm{~nm}$ & \multirow{2}{*}{1.35} & 4.84 & 0.95 & 31.35 & 5.90 \\
$\sigma=1.4 \%$ & & & 5.21 & 4.99 & 29.11 & 0.04 \\
bulk & - & \multirow{2}{*}{1.90} & 4.52 & 0.90 & 10.55 & 2.89 \\
$\sigma=1.9 \%$ & & & 6.55 & 2.01 & 108.4 & 0.0
\end{tabular}


неквадратичного отклонения порядка одного процента. Результаты вычислений приведены в табл. 2. В таблице приведены также параметры для диэлектрической проницаемости объемного образца (bulk). Эти значения получены в результате обработки дисперсионных кривых для диэлектрической проницаемости (действительной и мнимой части), полученных в работе [34] по результатам эллипсометрических измерений для монокристалла SBN61.

На рис. 6 приведены дисперсионные кривые для действительной $n$ (индекс рефракции) и мнимой $k$ (коэффициент экстинкции) частей показателя преломления. На рис. 7 представлены дисперсионные кривые для действительной и мнимой частей диэлектрической проницаемости. Здесь же приведены соответствующие кривые для объемного образца из [34]. Как видно из правой части рисунков, в длинноволновой части спектра $(E<3.5 \mathrm{eV})$, пленки 35 и 115 min практически прозрачны.

Количественно оптическая ширина запрещенной щели $E_{g}$ может быть оценена из спектральной зависимости соотношения $\left(\alpha^{\prime \prime} E\right)^{2}=\operatorname{const}\left(E-E_{g}\right)$ для прямых межзонных переходов, где коэффициент поглощения $\alpha^{\prime \prime}$ связан с мнимой частью показателя преломления следующим образом

$$
\alpha^{\prime \prime}=\frac{2}{\hbar c} k E .
$$

Аппроксимация графика зависимости $\left(\alpha^{\prime \prime} E\right)^{2}$ от энергии прямой линией в области резкого подъема поглощения дает значения 4.6 и $4.62 \mathrm{eV}$ для пленок SBN61 с временем напыления 35 и $115 \mathrm{~min}$ соответственно. Для монокристалла вычисления дают значение $4.31 \mathrm{eV}$.

\section{4. Обсуждение и выводы}

Гетроэпитаксиальные пленки SBN61 разной толщины нанесены эпитаксиально на монокристаллические подложки $\mathrm{MgO}$ (001). Рентгенодифракционные данные показали соосность пленок и подложки вдоль тетрагонального (001) направления. В плоскости подложки пленки ориентированы двояко: ось $[100]_{\mathrm{SBN}}$ пленки развернута на угол $\pm 18.43^{\circ}$ относительно оси $[100]_{\mathrm{MgO}}$. Разворот в плоскости, по мнению [19], происходит из-за того, что такое расположение пленки соответствует минимуму деформации, а эти две ориентации $\left( \pm 18.43^{\circ}\right)$ отвечают двум антифазным доменам. Объемный кристалл имеет параметры решетки $a_{0}=12.456 \AA, c_{0}=3.936 \AA$ [11]. Из наших измерений следует, что параметры решетки пленок 35 и $115 \mathrm{~min}$ равны $a=12.37 \AA, c=3.953 \AA$, т.е. пленки сжаты в плоскости подложки и вытянуты вдоль нормали к плоскости подложки. Для вынужденной деформации в плоскости пленки имеем $u_{m}=\left(a-a_{0}\right) / a_{0}=-0.7 \%$. Отметим, что объем элементарной ячейки пленки меньше, чем у объемного кристалла на 1\%. Аналогичное явление наблюдается в пленках твердых растворов титаната бария стронция при слоевом механизме роста [35]. Данные работы [28] и наши предварительные рентгеноструктурные исследования показали, что в пленках SBN61 при толщинах менее $150 \mathrm{~nm}$ наблюдается изменение параметров элементарной ячейки. Рассматриваемые пленки одинаково деформированы относительно объемного кристалла, поэтому интересен вопрос различия других свойств этих пленок, чем и был вызван выбор пленок этих толщин.

Выполнены измерения коэффициентов пропускания пленок в оптическом диапазоне, по которым определены параметры дисперсии диэлектрической проницаемости и показателя преломления. Дисперсионные кривые для пленок $35(202 \mathrm{~nm})$ и $115 \mathrm{~min}(736 \mathrm{~nm})$ подобны дисперсионным кривым объемного кристалла, однако имеют меньшее значение действительной части показателя преломления. Пленки имеют прозрачный вид без цветовых оттенков.

Как видно из спектров пропускания, край оптического поглощения сдвигается в область меньших энергий при увеличении толщины пленки, что должно соответствовать уменьшению ширины запрещенной зоны пленок. Однако вычисления по формуле Тауца (Tauc) не дают такой последовательности. Возможно, это связано с существованием в пленках сегнетоэлектрических доменов и структурных дефектов, нарушающих трансляционную периодичность, которая лежит в основе вывода формулы. Мы считаем, что более корректно, для оценки края поглощения и энергии перехода, характеризующего ширину запрещенной зоны, провести аппроксимацию спектров при учете в дисперсионной формуле для диэлектрической проницаемости только одного осциллятора. Энергия такого осциллятора будет соответствовать первому уровню энергии межзонного перехода, а минимальная энергия сечения на половине высоты пика мнимой части диэлектрической проницаемости будет качественно характеризовать ширину запрещенной зоны. В нашем случае такие вычисления дают значения энергии осцилляторов 4.67 и $4.40 \mathrm{eV}$ для пленок SBN61 с временем напыления 35 и $115 \mathrm{~min}$ соответственно. Для ширины запрещенной зоны из таких вычислений получим соответственно 4.61 и $4.39 \mathrm{eV}$. Из сравнения видно, что ширина (затухание) осциллятора может вносить существенные коррективы в величину запрещенной зоны.

Авторы выражают благодарность Л.И. Ивлевой за предоставление порошка SBN61, Л.А. Резниченко за помощь в изготовлении керамической мишени

\section{Список литературы}

[1] Ю.С. Кузьминов. Сегнетоэлектрические кристаллы для управления лазерным излучением. Наука, М. (1982). $400 \mathrm{c}$.

[2] P.V. Lenzo, E.G. Spencer, A.A. Ballman. Appl. Phys. Lett. 11, 23 (1967).

[3] A.M. Glass. J. Appl. Phys. 40, 4699 (1969).

[4] R.R. Neurgaonkar, W.K. Cory, J.R. Oliver. Ferroelectrics 51, 3 (1983). 
[5] R.R. Neurgaonkar, W.K. Cory, J.R. Oliver, M.D. Ewbank, W. Hall. Opt. Eng. 26, 392 (1987).

[6] P. Yeh, A.E.T. Chiou. Opt. Lett. 12, 138 (1987).

[7] J.E. Ford, Y. Fainman. J. Opt. Soc. Am. A 9, 1183 (1992).

[8] G.L. Wood, W.W. Clark, M.J. Miller, E.J. Sharp, G.J. Salano, R.R. Neurgaonkar. IEEE J. Quantum Electron 23, 2126 (1987).

[9] J.R. Carruthers, M. Grasso. J. Electrochem. Soc. 117, 1426 (1970).

[10] P.B. Jamieson, S.C. Abrahams, J.L. Bernstein. J. Chem. Phys. 48, 5048 (1968).

[11] Т.С. Черная, Б.А. Максимов, И.В. Верин, Л.И. Ивлева, В.И. Симонов. Кристаллография 42, 421 (1997).

[12] M.P. Trubelja, E. Ryba, D.K. Smith. J. Mater. Sci. 31, 1435 (1996).

[13] Т.С. Черная, Б.А. Максимов, Т.Р. Волк, Л.И. Ивлева, В.И. Симонов. ФТТ 42, 1668 (2000).

[14] S. Podlozhenov, H.A. Graetsch, J. Schneider, M. Ulex, M. Wöhlecke, K. Betzler. Acta Cryst. B 62, 960 (2006).

[15] T. Lukasiewicza, M.A. Swirkowicz, J. Dec, W. Hofman, W. Szyrski. J. Cryst. Growth 310, 1464 (2008).

[16] K. Dorywalski, B. Andriyevsky, C. Cobet, M. Piasecki, I.V. Kityk, N. Esser, T. Łukasiewicz, A. Patryn. Opt. Mater. 35, 887 (2013).

[17] А.Е. Андрейчук, Л.М. Дорожкин, Ю.С. Кузьминов, И.А. Масляницын, В.Н. Молчанов, А.А. Русаков, В.И. Симонов, В.Д. Шигорин, Г.П. Шипуло. Кристаллография 20, 1094 (1984).

[18] R.R. Neurgaonkar, W.F. Hall, J.R. Oliver, W.W. Ho, W.K. Cory. Ferroelectrics 87, 167 (1988)

[19] S. Schwyn Thöny, K.E. Youden, J.S. Harris jr., L. Hesselink. Appl. Phys. Lett. 65, 2018 (1994).

[20] C.M. Rouleau, G.E. Jellison jr., D.B. Beach. Appl. Phys. Lett. 82, 2990 (2003).

[21] M. Cuniot-Ponsard, J.M. Desvignes, B. Ea-Kim, E. Leroy. J. Appl. Phys. 93, 1718 (2003).

[22] Z.R. Shen, H. Ye, C.L. Mak, T.Y. Yum, K.H. Wong. Thin Solid Films 515, 3475 (2007).

[23] A. Infortuna, P. Muralt, M. Cantoni, A. Tagantsev, N. Setter. J. Eur. Cer. Soc. 24, 1573 (2004).

[24] Y.B. Yao, W.C. Liu, C.L. Mak, K.H. Wong, H.L. Tam, K.W. Cheah. Thin Solid Films 519, 52 (2010).

[25] Y.B. Yao, W.C. Liu, C.L. Mak, K.H. Wong. AIP Advances 1, 032172 (2011).

[26] M.K. Ryu, S.H. Lee, H.J. Joo, J.P. Kim, M.S. Jang, Y.S. Yang. Ferroelectrics 260, 99 (2001).

[27] M. Cuniot-Ponsard, J.M. Desvignes, B. Ea-Kim, E. Leroy. J. Appl. Phys. 93, 1718 (2003).

[28] Г.Н. Толмачев, А.П. Ковтун, И.Н. Захарченко, И.М. Алиев, А.В. Павленко, Л.А. Резниченко, И.А. Вербенко. ФТТ 57, 2050 (2015).

[29] O. Stenzel. The Physics of Thin Film Optical Spectra. An Introduction. Springer, Berlin (2005). 276 p.

[30] В.Б. Широков. Оптика и спектроскопия 112, 137 (2012).

[31] В.Б. Широков, Ю.И. Головко, В.М. Мухортов. ЖТФ 82, 79 (2012).

[32] Л.Д. Ландау, Е.М. Лифшиц. Электродинамика сплошных сред. ГИТТЛ, М. (1957). 532 с.

[33] S. Mamoun, A.E. Merad, L. Guilbert. Comput. Mater. Sci. 79, 125 (2013).

[34] K. Dorywalski, I. Maciejewski, T. Krzyżyński. Mechatronics 37, 33 (2016).

[35] В.М. Мухортов, Ю.И. Головко, С.В. Бирюков, А. Анохин, Ю.И. Юзюк. ЖТФ 86, 93 (2016). 